\title{
Matching the Budyko functions with the complementary evaporation relationship: consequences for the drying power of the air and the Priestley-Taylor coefficient
}

\author{
J.-P. Lhomme and R. Moussa \\ Correspondence to: Roger Moussa (roger.moussa@inra.fr)
}

The copyright of individual parts of the supplement might differ from the CC-BY 3.0 licence. 


\section{S.1 Properties of the function $E / P=B_{1}{ }^{\prime}\left(\Phi_{0}\right)$}

Putting $y=E / P$ with $0 \leqslant y \leqslant 1, x=\Phi_{0}$ and $a=\alpha_{0} /\left[(1+b) \alpha_{w}\right]$, function $B_{l}$ ' given by Eq. (20) can be rewritten as:

$10 x=a\left[\left(y^{-\lambda}-1\right)^{-1 / \lambda}+b y\right]$.

For $x=0, y$ is obviously equal to 0 . When $x$ tends to infinite the result is less evident. Eq. (S1.1) can be rewritten as:

$\frac{x}{a}-b y=\frac{1}{\left(\frac{1}{y^{\lambda}}-1\right)^{\frac{1}{\lambda}}}$.

When $x$ tends to infinite, given that $y$ is limited by 1, the right-hand term of the equation should tend to infinite. This means that $y$ should tend to $l$ so that the denominator tends to zero.

15

The derivative of the function (Eq. S1.1) is given by:

$\frac{d x}{d y}=a\left[b+y^{-(\lambda+1)}\left(y^{-\lambda}-1\right)^{-\frac{1}{\lambda}-1}\right]$,

which can be rewritten as:

$\frac{d y}{d x}=\frac{1}{a}\left[b+\left(1-y^{\lambda}\right)^{-(1+\lambda) / \lambda}\right]^{-1}$.

Close to $x=0, y$ is close to zero and the derivative can be approximated by:

$20 \frac{d y}{d x} \approx \frac{1}{a(1+b)}\left[1-\left(\frac{1+\lambda}{\lambda(1+b)}\right) y^{\lambda}\right] \approx \frac{\alpha_{w}}{\alpha_{0}}$.

If Eq. (22) is taken into account:

$\frac{\alpha_{w}}{\alpha_{0}}=\frac{\left[1+b\left(1+x^{\lambda}\right)^{-\frac{1}{\lambda}}\right]}{(1+b)}$,

which means that $\alpha_{w} / \alpha_{0}$ and $d y / d x$ tend to $l$ when $x$ tends to zero. 


\section{S.2 Properties of the function $E / E_{0}=B_{2}{ }^{\prime}\left(\Phi_{0}^{-1}\right)$}

With $X=\Phi_{0}{ }^{-1}, Y=E / E_{0}$ and the parameter $a$ defined as above in $S 1$, function $B_{2}{ }^{\prime}$ (Eq. 21) can be written as

$X^{-\lambda}=Y^{-\lambda}-\left(\frac{1}{a}-b Y\right)^{-\lambda}$.

When $X$ tends to zero, $Y$ (limited by 1 ) necessarily tends to zero, and when $X$ tends to infinite $Y$ tends to $1 /[(1+b) a]=\alpha_{w} / \alpha_{0}$,

5 which is equal to 1 according to Eq. (S1.6) $(x=1 / X=0)$.

The derivative of $B_{2}$ ' can be written as:

$\frac{d Y}{d X}=\frac{X^{-\lambda-1}}{Y^{-\lambda-1}+b\left(\frac{1}{a}-b Y\right)^{-\lambda-1}}=\frac{1}{\left(\frac{X}{Y}\right)^{\lambda+1}\left[1+\frac{b}{\left(\frac{1}{a Y}-b\right)^{\lambda+1}}\right]}$.

When $X$ tends to zero, $Y$ also tends to zero and the term into square brackets tends to $l$ which means that:

$\frac{d Y}{d X} \rightarrow\left(\frac{Y}{X}\right)^{\lambda+1}$.

10 Taking into account Eq. (S2.1), we have:

$\left(\frac{Y}{X}\right)^{\lambda+1}=\left[1-\left(\frac{1}{a Y}-b\right)^{-\lambda}\right]^{\frac{\lambda+1}{\lambda}}$,

which tends to $l$.

S.3 Transcendental forms of the basic equations $E / P=B_{I}\left(\Phi_{p}\right)$ and $E / E_{p}=B_{2}\left(\Phi_{p}^{-1}\right)$

15 Eqs. (4) and (5) have the same following form:

$y=\left(1+x^{-\lambda}\right)^{-1 / \lambda}$,

with $x=\Phi_{p}$ and $y=E / P$. Eq. (S3.1) can be also written as:

$x=\left(y^{-\lambda}-1\right)^{-1 / \lambda}$.

With similar notations, Eq. (23) can be written as:

$20 y+\left(y^{-\lambda}-1\right)^{-1 / \lambda}=x+\left(1+x^{-\lambda}\right)^{-1 / \lambda}$.

Eq. (S3.3) is equivalent to $y+x=x+y$, which means that $\mathrm{S} 3.1$ or S3.2 are solutions of Eq. (S3.3).

A similar reasoning can be conducted with Eq. (24), which can be written with $X=\Phi_{p}^{-1}$ and $Y=E / E_{p}$ :

$\left[1-Y+\left(1+X^{-\lambda}\right)^{-1 / \lambda}\right]^{-\lambda}=Y^{-\lambda}-X^{-\lambda}$.

Given that Eq. (S3.1) is verified by $X$ and $Y$ :

$25 Y^{-\lambda}=1+X^{-\lambda}$.

Eq. (S3.4) is equivalent to $1=1$, which means that Eq. (S3.1) or (S3.2) is solution of Eq. (S3.4). 


\section{S.4 Calculations made with the Fu-Zhang equation}

The Fu-Zhang equation is written as:

$\frac{E}{P}=1+\Phi_{p}-\left[1+\left(\Phi_{p}\right)^{\omega}\right]^{\frac{1}{\omega}}$.

First, we study the feasible domain of the drying power of the air $E_{a}$ and the correspondence with the evaporation rate $E$.

5 Inserting Eq. (S4.1) into Eq. (9) yields:

$\frac{E_{a}}{E_{P}}=D\left(\Phi_{P}^{-1}\right)=\left(1+\frac{\Delta}{\gamma}\right)\left(1-\frac{1}{(1+b) \alpha_{w}}\left\{1+b\left[1+\Phi_{P}^{-1}-\left(1+\Phi_{P}^{-\omega}\right)^{\frac{1}{\omega}}\right]\right\}\right)$.

The limits given in Eqs. (11), (12) and (13) are independent from the Budyko function used. Consequently $D^{*}$ remains unchanged:

$D^{*}=\frac{b}{(1+b) \alpha_{w}}\left(1+\frac{\Delta}{\gamma}\right)$.

10 Using a similar reasoning as in Eqs (14), (15), (16) and (17), we obtain:

$d^{*}=2^{\frac{1}{\omega}}-1$,

$\omega=\frac{\ln 2}{\ln \left(d^{*}-1\right)}$,

$\delta^{*}=\left(1+\frac{\Delta}{\gamma}\right) \frac{b}{(1+b) \alpha_{w}}\left(1-2^{-\frac{1}{\lambda}}\right)=D^{*} d^{*}$.

Second, we link the Priestley-Taylor coefficient $\alpha_{0}$ to the Fu-Zhang shape parameter $\omega$. Substituting $E_{p}$ in Eq. (S4.1)

15 by its value given by Eq. (18) and putting $\Phi_{0}=E_{0} / P$ gives:

$\frac{E}{P}=1+\frac{(1+b) \alpha_{w}}{\alpha_{0}} \Phi_{0}-b \frac{E}{P}-\left\{1+\left[\frac{(1+b) \alpha_{w}}{\alpha_{0}} \Phi_{0}-b \frac{E}{P}\right]^{\omega}\right\}^{\frac{1}{\omega}}$.

Eq. (S4.7) can be rewritten as:

$\left[1+(1+b)\left(\frac{\alpha_{w}}{\alpha_{0}} \Phi_{0}-\frac{E}{P}\right)\right]^{\omega}=1+\left[(1+b) \frac{\alpha_{w}}{\alpha_{0}} \Phi_{0}-b \frac{E}{P}\right]^{\omega}$.

An equation similar to Eq. (21) can be obtained expressing $E / E_{0}$ as a function of $\Phi_{0}^{-1}=P / E_{0}$ :

$20\left[1+\frac{(1+b)}{\Phi_{0}^{-1}}\left(\frac{\alpha_{w}}{\alpha_{0}}-\frac{E}{E_{0}}\right)\right]^{\omega}=1+\left(\frac{1}{\Phi_{0}^{-1}}\right)^{\omega}\left[\frac{(1+b) \alpha_{w}}{\alpha_{0}}-b \frac{E}{E_{0}}\right]^{\omega}$.

Eqs. (S4.8) and (S4.9) obtained from the Fu-Zhang formulation correspond respectively to $E / P=B_{1}{ }^{\prime}\left(\Phi_{0}\right)$ (Eq. 20) and $E / E_{0}$ $=B_{2}{ }^{\prime}\left(\Phi_{0}^{-1}\right)$ (Eq. 21) obtained with the Turc-Mezentsev equation.

Using a similar reasoning as in Eq. (22), the expression of $\alpha_{0}$ can be inferred by matching Eqs. (S4.8) and (S4.1): for a given value of the aridity index $\Phi$, we have the same value of $E / P$. This leads to:

$25\left\{(1+b)\left[\left(\frac{\alpha_{w}}{\alpha_{0}}-1\right) \Phi+\left(1+\Phi^{\omega}\right)^{\frac{1}{\omega}}\right]-b\right\}^{\omega}=1+\left\{\left[\frac{(1+b) \alpha_{w}}{\alpha_{0}}-b\right] \Phi+b\left(1+\Phi^{\omega}\right)^{\frac{1}{\omega}}-b\right\}^{\omega}$.

Eq. (S4.10) is equivalent to Eq. (22), but with a transcendental form. It can be resolved numerically and Fig. (S1) shows the variation of the Priestley-Taylor coefficient $\alpha_{0}$ as a function of the aridity index $\Phi$ for different values of the $\omega$ parameter. The shape of the curves is very similar to those of Fig. (5a) obtained with the parameter $\lambda$ of the Turc-Mezentsev function. 


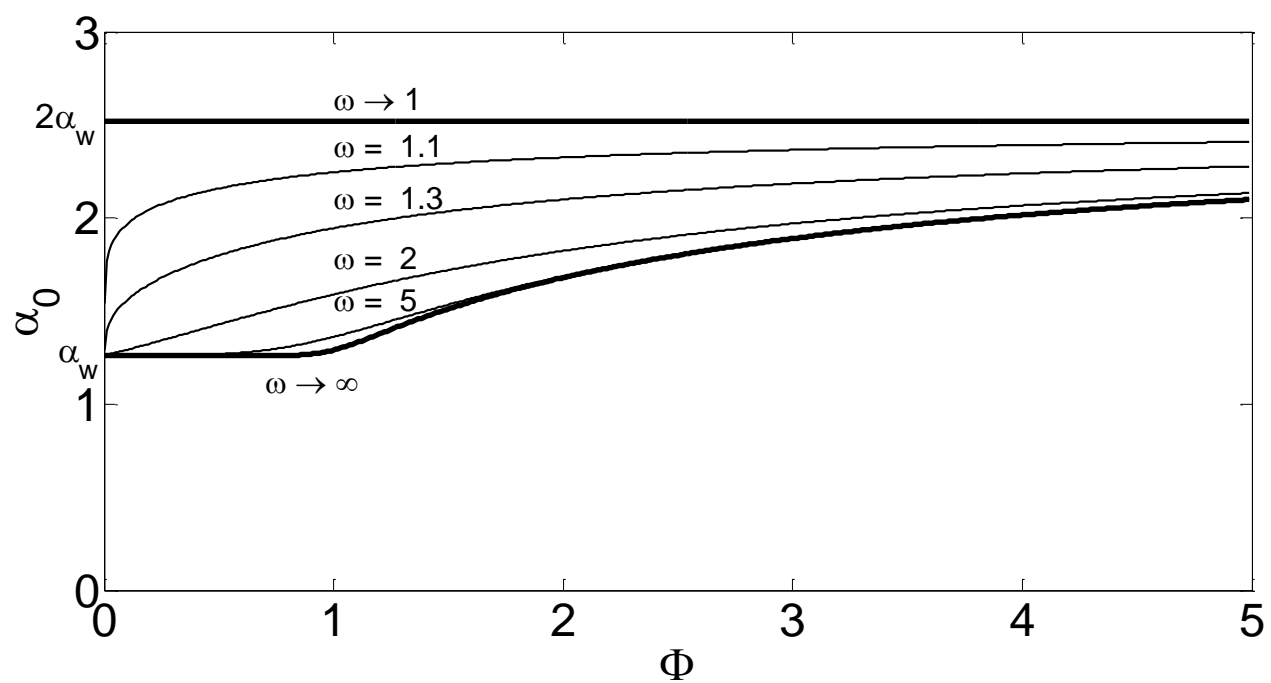

Figure S1: Variation of the Priestley-Taylor coefficient $\alpha_{0}$ with $b=1$ as a function of the aridity index $\Phi$ for different values of the 5 shape parameter $\omega$ of the Fu-Zhang function. The bold lines indicate the limits of the feasible domain. 
S.5 Results obtained with the Turc-Mezentsev function making $b=4.5$ instead of $b=1$

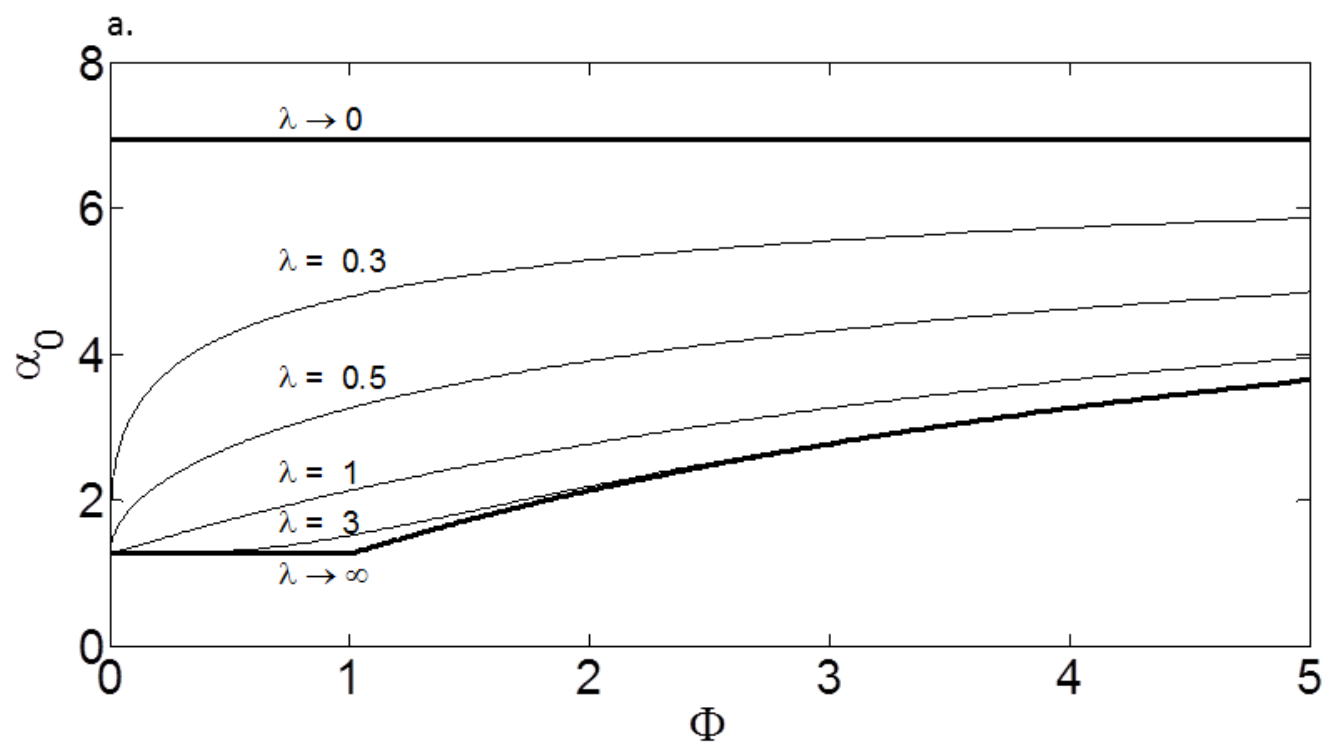

b.

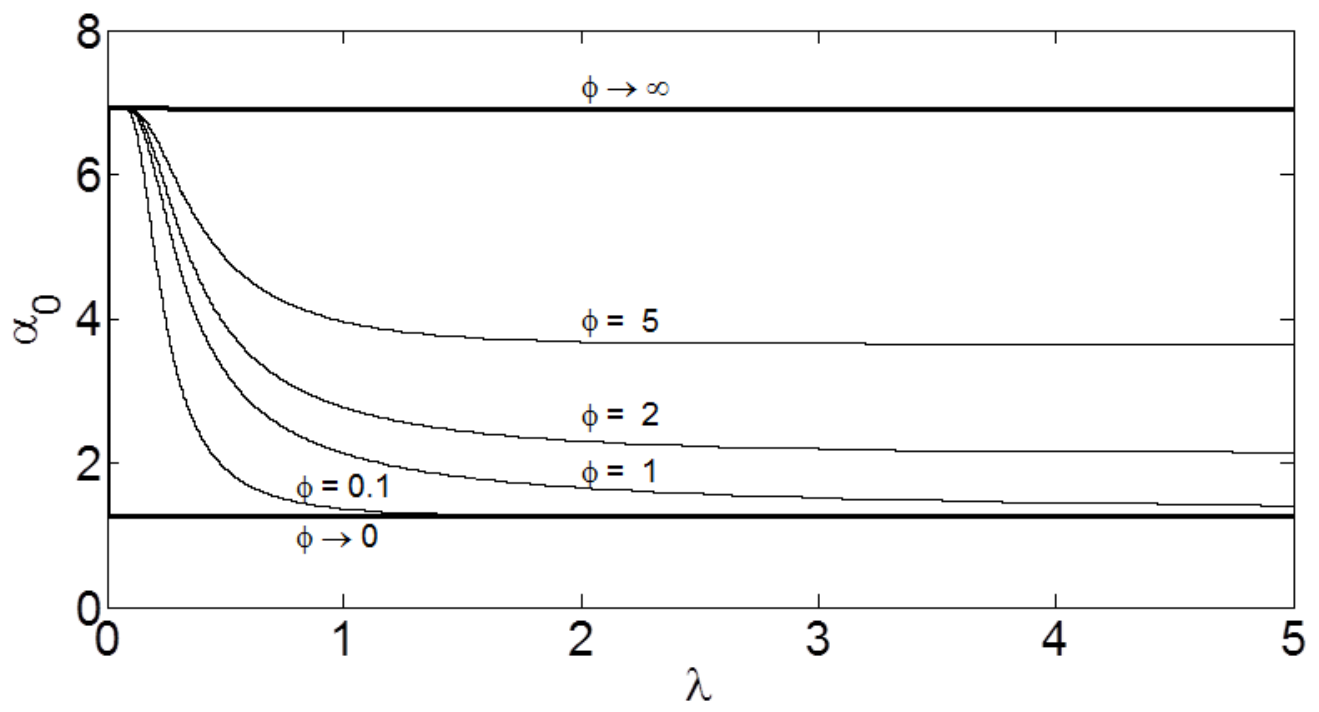

Figure S2: Variation of the Priestley-Taylor coefficient $\alpha_{0}$ (Eq. (22) with $b=4.5$ and $\alpha_{w}=1.26$ ): (a) as a function of the aridity index $5 \Phi$ for different values of the shape parameter $\lambda$ of the Turc-Mezentsev function; (b) as a function of $\lambda$ for different values of the aridity index $\Phi$. The bold lines indicate the limits of the feasible domain. 